\title{
Relación clínica y ultrasonográfica de lesiones del manguito rotador en una población del DIF Aguascalientes
}

Ramírez-Cervantes José de Jesús*, Arriaga-Rivera Javier**

\begin{abstract}
Resumen
- Objetivo: Determinar la correlación de las pruebas clínicas - versus ultrasonido del músculo esquelético en las lesiones - del manguito rotador (LMR). Métodos: Se realizó un estudio - descriptivo de corte transversal, en el cual se seleccionaron - los expedientes con diagnóstico de LMR, del periodo de oc- tubre del 2017 a febrero del 2018; se tomó en cuenta

- la edad, género, tipo de actividad, comorbilidades, pruebas

- clínicas (positivas) y hallazgos ultrasonográficos. Posterior-

- mente, se realizó el análisis estadístico de la correlación de

- variables con P de Pearson. Resultados: Se obtuvo una media

- de edad de 59 años, la correlación de P de Pearson para las

- pruebas clínicas fueron: supraespinoso-USG de 0.860, pin-

- zamiento subacromial-USG 0.269, subescapular-USG 0.505

- y para la tendinopatía bicipital-USG 0.25I; la correlación

- de la pruebas clínicas-USG global fue de 0.625. Conclusión:

- este estudio revela una moderada correlación de las prue-

- bas clínicas-USG, por lo que el ultrasonido es un adecuado

- medio de complementación diagnóstica. LUXMÉDICA, AÑO 13,

- NÚMERO 38, MAY-AGO 2018PP 19-26.
\end{abstract}

Palabras clave: manguito rotador, ultrasonido, correlación, tendinopatía, pinzamiento.

\section{Abstract}

Objective: To look for a significant association of the clinical tests with the ultrasonographic findings in the patients with rotator cuff injury (RCl). Methods: A cross-sectional descriptive study was carried out; medical files from October-November 2017 were selected; age, sex, type of activity, comorbidities, clinical tests (positive), and ultrasonographic findings were considered. Subsequently, the statistical analysis of the Pearson correlation coefficient was performed. Results: A mean age of 64 years was obtained, Pearson correlation for clinical tests were: supraspinatus-USG of 0.416 , subacromial impingement-USG 0.673, subscapular-USG 0.628, and for biceps tendinopathy-USG 0.924; global correlation of clinical tests-USG was 0.622. Conclusion:This study reveals a moderate correlation of clinical tests-USG; therefore, ultrasound is an adequate diagnostic complementary test. LUXMÉDICA, AÑo 13, NÚMERO 38, MAY-AG0 2018 PP 19-26.

\section{Introducción}

Las lesiones del manguito rotador (LMR) son una causa frecuente de consulta en los servicios de medicina de rehabilitación, debido al dolor y la discapacidad que genera. Se estima que la incidencia es de 3.7 casos de 100,000 por año, con mayor frecuencia en la quinta década de la vida. ${ }^{1-3}$ En trabajadores que desempeñan actividades de carga, suele incrementarse hasta en un $18 \% .^{1}$

\footnotetext{
* Médico Pasante de Servicio Social de la Universidad Cuauhtémoc Aguascalientes

** Médico Especialista en Rehabilitación, Alta especialidad en Rehabilitación Pediátrica Centro de Rehabilitación e Inclusión Social, DIF Aguascalientes.

Fecha de recibido: 15 de enero 2018

Fecha de aceptación: 5 de marzo 2018

Correspondencia: Dr. Javier Arriaga Rivera, Centro de Rehabilitación e Inclusión Social del DIF Estatal Aguascalientes. Avenida Mahatma Gandhi sln esq. República de Paraguay Colonia Agricultura, Código postal 20234, Aguascalientes, Ags., México. Teléfono 0144991760 00, movimedic-ags@ hotmail.com.
} 
Estas lesiones producen dolor persistente con duración variable de seis semanas en el $70 \%$ de los pacientes y hasta seis meses en $46 \%$, limitando las actividades de vida diaria y la capacidad para laborar durante un largo periodo, disminuyendo así su productividad. En países desarrollados, se ha documentado un gasto de 326 euros por paciente para la atención de este problema a lo largo de seis meses y un promedio de 2069 euros por gastos indirectos durante el mismo tiempo, es decir, un costo aproximado de 47 mil pesos en promedio. ${ }^{4,5}$

En el centro de Desarrollo Integral de la Familia (DIF) Aguascalientes, representa el $9.8 \%$ de los motivos de atención en la consulta de rehabilitación y los principales problemas que se mencionan son dolor y limitación de las actividades de vida diaria y laborales.

Los factores que suelen asociarse con el desarrollo de la patología, van desde el tipo de actividad que realiza la persona ${ }^{6}$ (actividades de carga por arriba de hombros) hasta factores personales como: diabetes mellitus (DM), obesidad, tabaquismo, dislipidemias, factores genéticos y anatómicos. ${ }^{1,7}$

Actualmente, existen diversas pruebas diagnósticas (clínicas e imagen) que cuentan con una variabilidad en su sensibilidad (S) y especificidad (E), colocando a la resonancia magnética (RM) como el estándar de oro (S: 100\%) usado para el diagnóstico en países desarrollados; $;, 9$ sin embargo, por accesibilidad y costos para nuestro medio, las pruebas clínicas y el ultrasonido (S: 90\%) siguen siendo los métodos más utilizados..$^{9-11}$ El objetivo del presente estudio fue determinar la correlación de las pruebas clínicas versus ultrasonido del músculo esquelético en las lesiones del manguito rotador en pacientes atendidos en el DIF Aguascalientes.

\section{| | | | | | | | | | | | | | | | | | | | | | | | | | | | | | | | | | | | | | | | | | | | | | | | | | | | | | | | | | | | | | | | | | | | | | | | | | | | | | | | | | | | | | | | | | | | | | | | | | | | | | | | | | | | | | | | | | |}

\section{Material y métodos}

Se realizó un estudio descriptivo de corte transversal, para el cual se seleccionaron los expedientes de los pacientes evaluados en consulta de medicina de rehabilitación en el Desarrollo Integral de la Familia (DIF) Estatal de Aguascalientes de octubre de 2017 a febrero de 2018. Las variables estudiadas fueron: la evaluación por el médico especialista en medicina de rehabilitación, el diagnóstico de la lesión del manguito rotador, descripción de pruebas clínicas realizadas (Jobe y brazo caído para supraespinoso, lift off o subescapular para el músculo subescapular, Neer y Hawkin para pinzamiento subacromial, Speed y Yerga- son para la valoración de la porción larga del bíceps, prueba del infraespinoso y del redondo menor para los mismos), descripción de estudio ultrasonográfico realizado por médico radiólogo, edad entre 18 y 90 años. Criterios de exclusión: pacientes previamente tratados por otros servicios, con infiltraciones previas, antecedentes previos de cirugía de hombro y de fracturas de clavícula-humero. Criterios de eliminación: expediente incompleto o sin nota de medico especialista.

Se realizó una base de datos en Excel 2013, en donde se tomaron en cuenta la edad, género, tipo de actividad, comorbilidades, pruebas clínicas positivas y negati- 
vas (anteriormente descritas), así como los hallazgos ultrasonográficos reportados por el médico radiólogo. Posteriormente, los datos se exportaron al programa estadístico IBM SPSS versión 23 para Windows, para su análisis estadístico. Se utilizó la $p$ de Pearson para correlacionar las pruebas clínicas y los hallazgos ultrasonográficos, posteriormente se clasificó la correlación de las variables con los rangos previamente ya establecidos $( \pm 0.96$ a \pm 1.0 perfecta, \pm 0.85 a \pm 0.95 fuerte, \pm 0.70 a \pm 0.84 significativa, \pm 0.50 a 0.69 modera$\mathrm{da}, \pm 0.20$ a \pm 0.49 débil, \pm 0.10 a \pm 0.19 muy débil, \pm 0.0 a \pm 0.09 nula). Se obtuvo la aceptación por el Comité de Bioética de la institución DIF Estatal de Aguascalientes para la realización del presente estudio.

\section{|| || |||||||||||||||||||||||||||||||||||||||||||||||||||||||||||||||||||||||||||||||||||||||||||||||||||||||||||||||||||}

\section{Resultados}

De un total de 1180 pacientes valorados durante el periodo previamente señalado, en la Consulta de Rehabilitación, se seleccionaron aquellos expedientes de pacientes con diagnóstico de $L M R$, obteniéndose 63 expedientes en total, de los cuales 35 cumplieron con los criterios de inclusión, 24 se excluyeron y 4 expedientes fueron eliminados.

El promedio de edad de los pacientes fue de 59 años (22-90 años), el 20\% fueron hombres y el $80 \%$ mujeres. En relación a la ocupación, el $57.1 \%$ realizaba actividades domésticas, el $8.6 \%$ actividades de carga y el $34.3 \%$ actividades sin carga; en relación a las comorbilidades, el $60 \%$ de las personas se reportaron como sanas, mientras que el $25.7 \%$ presentó DM, $14.3 \%$ hipertensión arterial sistémica (HAS), 5.8\% presentó hipotiroidismo, y $2.9 \%$ secuelas de EVC, así como neumopatía crónica.

La lesión más frecuente reportada en la clínica fue la lesión del supraespinoso en el $85.7 \%$ de los pacientes, seguido de lesión del subescapular y pinzamiento, teniendo el 60 y $39.9 \%$ de los casos respectivamente. El $48.5 \%$ de los casos presentó una tendinopatía bicipital asociada. El $82.8 \%$ de los pacientes tuvo una lesión múltiple (dos o más lesiones clínicas), mientras que solo el $17.2 \%$ reportó lesión de un solo músculo (tabla 1 y 2 ).

\section{Tabla I}

Tipo de lesión en población con diagnóstico de lesión del manguito rotador en pacientes del DIF Aguascalientes.

\begin{tabular}{|ll|}
\hline Lesión Clínica & Porcentaje \\
\hline Supraespinoso & 85.7 \\
\hline Subescapular & 60 \\
\hline Tendinopatía bicipital & 48.5 \\
\hline Pinzamiento subacromial & 39.9 \\
\hline
\end{tabular}




\section{Tabla 2}

\section{Pruebas clínicas en pacientes con diagnóstico de lesión del manguito rotador en pacientes del DIF Aguascalientes.}

\begin{tabular}{|lcc|}
\hline Descripción Clínica de las lesiones. & Frecuencia & Porcentaje \\
\hline Jobe/Brazo caído. & 4 & 11.4 \\
\hline Subescapular. & 3 & 8.6 \\
\hline Jobe/Brz Caído + Neer/Hawkin. & 4 & 11.4 \\
\hline Jobe/Brz Caído + Subescapular. & 3 & 8.6 \\
\hline Jobe/Brz Caído + Speed/Yergarson. & 4 & 11.4 \\
\hline Jobe/Brz Caído + Speed/ Yergarson + Subescapular + Neer/Hawkin. & 41.4 \\
\hline Jobe/Brz Caído + SBE + Neer/Hawkin. & 4 & 5.7 \\
\hline SBE + Neer. & 2 & 5.7 \\
\hline Speed/Yergarson + Jobe/Brz Caído + Subescapular. & 2 & 20.0 \\
\hline Speed/Yergarson + Jobe/Brz Caído + Neer/Hawkin. & 7 & 5.7 \\
\hline Total & 2 & 100.0 \\
\hline
\end{tabular}

Por ultrasonido, la lesión del supraespinoso fue reportada en el $85.8 \%$ de los casos, mientras que el pinzamiento subacromial en un $37.3 \%$, la tendinopatía bicipital $45.9 \%$ y la lesión del subescapular en el $20.1 \%$. En el $71.4 \%$ de los pacientes, se reportó una lesión múltiple, mientras que el $28.6 \%$ solo tuvo lesión de una estructura (tabla 3).
La correlación de variables con la prueba $p$ de Pearson en forma global para las pruebas clínicas-USG fue de 0.625, mientras que en forma aislada las pruebas para supraespinoso-USG fueron de 0.860 , pinzamiento subacromial-USG 0.269 , subescapular-USG 0.505, y tendinopatía bicipital-USG 0.251 (tabla 4).

\section{Tabla 3}

\section{LMR por USG en pacientes con diagnóstico de lesión del manguito rotador en pacientes del DIF Aguascalientes.}

\begin{tabular}{|lcc|}
\hline Lesión del Manguito Rotador & No. de Casos & Porcentaje \\
\hline Lesión del supraespinoso & 7 & 20.0 \\
\hline Pinzamiento subacromial & 1 & 2.9 \\
\hline Tendinopatía bicipital & 2 & 5.7 \\
\hline Tendinopatía bicipital + lesión del supraespinoso & 8 & 22.9 \\
\hline Lesión del supraespinoso + pinzamiento subacromial & 6 & 17.1 \\
\hline Tendinopatía bicipital + lesión del supraespinoso + pinzamiento subacromial & 3 & 8.6 \\
\hline $\begin{array}{l}\text { Tendinopatía bicipital + lesión del supraespinoso + pinzamiento subacromial } \\
+ \text { lesión del subescapular }\end{array}$ & 1 & 2.9 \\
\hline Lesión del supraespinoso + lesión del subescapular & 4 & 11.4 \\
\hline Tendinopatía bicipital + pinzamiento subacromial & 1 & 2.9 \\
\hline Lesión del supraespinoso + lesión del subescapular + pinzamiento subacromial & 1 & 2.9 \\
\hline Tendinopatía bicipital & 1 & 2.9 \\
\hline Total & 35 & 100.0 \\
\hline
\end{tabular}




\section{Tabla 4}

Correlación de Pearson clínico-ultrasonográfica.

\begin{tabular}{|lc|}
\hline Maniobras/USG & Correlación de Pearson \\
\hline Tendinopatía bicipital/ USG & 0.251 \\
\hline Lesión del subescapular/USG & 0.505 \\
\hline Pinzamiento subacromial/USG & 0.269 \\
\hline Lesión del supraespinoso/USG & 0.860 \\
\hline Diagnóstico clínico / diagnóstico USG & 0.625 \\
\hline
\end{tabular}

\section{Discusión}

\section{Variables demográficas}

En este estudio, la edad media de presentación de LMR fue de 59 años, resultando similar a lo reportado por Micheroli, ${ }^{12}$ y Naredo, ${ }^{13}$ con una media de 50 años y lesión de espesor total del manguito rotador en aquellas personas $>65$ años, ${ }^{12}$ lo que nos indica que la edad se correlaciona con la severidad de la lesión. ${ }^{14}$

Reforzando este argumento, Forthom$\mathrm{me}^{15}$ reporta que existen diferencias significativas en la fuerza, medida con dinamómetro isocinético para los músculos del hombro en jóvenes, quienes desarrollan un $40 \%$ más de torque en comparación con adultos mayores, y existe diferencia de un $11 \%$ de fuerza en relación al lado dominante, factor que no fue tomado en cuenta para su análisis en este estudio. Además, se ha documentado que existe un desbalance muscular del trapecio y serrato (estabilizadores de escápula) asociado con LMR, con bajos torques a velocidades bajas y pobre reclutamiento mediante electromiografía (EMG). ${ }^{16}$ Varios autores refieren que la relación alterada de agonistas/antagonistas en hombro es precursora de lesión; de tal forma que, el supraespinoso al ser un elemento activo en la movilidad del hombro, requiere de estabilizadores como el deltoides y el complejo osteoligamentario coracoacromial para lograr una adecuada mecánica. ${ }^{17-21}$
En cuanto a la distribución por sexo, obtuvimos una frecuencia mayor en mujeres $(80 \%)$, que concuerda con los datos de Micheroli, ${ }^{12}$ mientras que Kumath ${ }^{22}$ hace referencia a lo contrario, teniendo mayor porcentaje en el sexo masculino (63\%), sin embargo, no se menciona cuál fue el motivo de una mayor prevalencia en hombres. Para nuestro estudio, los resultados se interpretan considerando que, la edad y el tipo de actividad doméstica que realizan las mujeres, ofrece un trauma repetitivo al hombro, asociado con los cambios degenerativos, que condicionan el desarrollo de la patología. ${ }^{13,23}$ Los estudios consultados involucran a hombres y mujeres para evaluación del pico de torque y trabajo; sin embargo, no se establecen diferencias significativas por sexo. ${ }^{21}$

\section{Comorbilidades asociadas}

Titchener et $\mathrm{al}^{24}$ reportan que las comorbilidades y factores de riesgo asociadas a LMR son la diabetes mellitus tipo 2 (DM), obesidad, sobrepeso, ingesta de corticoesteroides, entre otras, coincidiendo con lo reportado en este estudio donde la DM se reportó en $25.7 \%$ de los casos. En los pacientes con DM, dichos factores de riesgo se deben a la acumulación en los tendones de subproductos metabólicos de la glicación no enzimática ${ }^{25,26}$ y a depósitos de cristales de calcio en la matriz, los cuales se fusionan para formar grandes focos de calcificación, predisponiendo a otras lesiones. ${ }^{26,27}$ En relación a comorbilidades mús- 
culo esquelético como tendinitis de Quervain, epicondilitis lateral, dedos en gatillo y tendinitis aquílea, este estudio no reportó asociación de estas alteraciones, a diferencia de otros autores. ${ }^{24}$

\section{Ocupación}

En cuanto a la ocupación, este estudio reportó mayor incidencia de LMR en personas que realizan actividades domésticas $(57.1 \%)$ en comparación con cargadores (8.6\%). A diferencia de nuestros resultados, Silverstein ${ }^{28}$ reportó alta incidencia en trabajadores de carga, sin embargo Seidler ${ }^{29}$ menciona que la ocupación no tiene relación con LMR. Esta variabilidad en los resultados puede deberse al tipo de población estudiada y deben considerarse los factores como el trauma repetitivo o de alto impacto, así como las actividades por encima del hombro a razón de dosis respuesta como condicionantes del desarrollo de las lesiones.

\section{Tipos de lesión}

En este estudio la LMR más frecuente fue la del supraespinoso ( $85.8 \%$ de los casos), dicho resultado se compara con los obtenidos por Kumath, ${ }^{22}$ Naredo $^{13}$ y Kim, ${ }^{23}$ obteniendo hallazgos de lesión en múltiples estructuras del hombro y relacionándose con los estadios descritos anteriormente por Neer, ${ }^{30}$ comportamiento que se identificó en los pacientes del presente trabajo, donde el $71.4 \%$ tuvo una lesión múltiple, aunque no se clasificó acorde a los estadios de Neer. Por otra parte, la tendinopatía bicipital en el estudio realizado por Neviaser $^{31}$ fue reportada en $85 \%$ de los casos, difiriendo con nuestro estudio donde tuvo una incidencia del $45.9 \%$, como alteración asociada a LMR. Esta lesión puede explicarse debido a que el tendón del bíceps y la bursa subacromial-subdeltoidea están expuestas a fuerzas mecánicas que contribuyen al choque del manguito rotador o cuando existe un desgarro de éste, se comunica la articulación glenohumeral y la bursa subacromial-subdeltoidea, provocando inflamación y daño al tendón del bíceps, ya que la vaina sinovial de bíceps es una extensión de la membrana sinovial glenohumeral. ${ }^{13}$

El pinzamiento subacromial fue la segunda lesión más frecuente, después del supraespinoso, reportada en forma clínica en el $39.9 \%$ de los casos y por USG en $37.3 \%$. Dichos resultados concuerdan con Micheroli ${ }^{12}$ y Naredo, ${ }^{13}$ quienes reportaron esta lesión como una de las más frecuentes asociadas a LMR; sin embargo, no se establece un mecanismo claro y sólo se hace referencia a que puede variar dependiendo de la población en estudio, ${ }^{23}$ por lo que resulta relevante, para futuras investigaciones, determinar la causa o asociación. Por otra parte, las lesiones del infraespinoso y del redondo menor no se reportaron en forma clínica o por ultrasonido, resultado que contrasta con lo reportado por Taneja y colaboradores, ${ }^{32}$ encontrando lesiones tendinosas y musculares del infraespinoso en el $50 \%$ de los casos y del redondo menor en $19 \%$, mediante estudios de resonancia magnética en una población más joven (38 años), únicamente describiendo como factores etiológicos, movimientos rotacionales y trauma directo en algunos casos.

\section{Correlación de variables}

En el análisis estadístico obtenido con base en el coeficiente de correlación de variables $p$ de Pearson, demostró una moderada correlación de la clínica-USG global $(P=0.625) \quad e$ individual; supraespinoso $(P=0.860)$, pinzamiento subacromial $(P=0.269)$, subescapular $(P=0.505)$, tendinopatía bicipital $(P=0.251)$, dichas correlaciones, difieren de los resultados obtenidos por Micheroli, ${ }^{12}$ quien mostró un asociación débil para la mayoría de las variables. Cabe destacar que existe una gran cantidad de pruebas clínicas que pueden 
utilizarse, las cuales tienen una sensibilidad y especificidad variable (tabla 5),1,10,33 lo que puede limitar la detección de lesiones en forma clínica, además de los diversos protocolos de estudio por USG, ya que los resultados son operador-dependientes.13 Además, este autor tuvo una población más joven (media de 54 años) y una can- tidad mayor de pacientes en comparación con nuestro estudio. También debe tomarse en cuenta que un gran porcentaje de pacientes presenta lesiones complejas, por lo que algunas pruebas pueden no ser ideales en etapas agudas debido al dolor, dificultando la localización de la lesión.

\section{Tabla 5}

Sensibilidad y especificidad de las pruebas clínicas.

\begin{tabular}{|c|c|}
\hline Prueba & Valor Diagnóstico (\%). \\
\hline \multicolumn{2}{|c|}{ Subescapular } \\
\hline Subescapular/Life off & $\begin{array}{l}\text { Sensibilidad: } 17-100 \\
\text { Especificidad: } 60-98\end{array}$ \\
\hline Belly press & $\begin{array}{l}\text { Sensibilidad: } 40-43 \\
\text { Especificidad: } 93-98\end{array}$ \\
\hline Belly off & $\begin{array}{l}\text { Sensibilidad: } 14-86 \\
\text { Especificidad: } 91-95\end{array}$ \\
\hline Bear hug & $\begin{array}{l}\text { Sensibilidad: } 60 \\
\text { Especificidad: } 92\end{array}$ \\
\hline \multicolumn{2}{|c|}{ Infraespinoso } \\
\hline Prueba del infraespinoso/External rotation lag sign & $\begin{array}{l}\text { Sensibilidad: } 46-98 \\
\text { Especificidad: } 72-98\end{array}$ \\
\hline \multicolumn{2}{|c|}{ Supraespinoso } \\
\hline Jobe & $\begin{array}{l}\text { Sensibilidad: } 53-89 \\
\text { Especificidad: } 65-82\end{array}$ \\
\hline Brazo caído & $\begin{array}{l}\text { Sensibilidad: } 10-73 \\
\text { Especificidad: } 77-98\end{array}$ \\
\hline \multicolumn{2}{|c|}{ Pinzamiento subacromial } \\
\hline Neer & $\begin{array}{l}\text { Sensibilidad: } 68-89 \\
\text { Especificidad: } 49-98\end{array}$ \\
\hline Hawkin & $\begin{array}{l}\text { Sensibilidad: } 71-92 \\
\text { Especificidad: } 44-78\end{array}$ \\
\hline \multicolumn{2}{|c|}{ Tendinopatía Bicipital. } \\
\hline Speed & $\begin{array}{l}\text { Sensibilidad: } 53 \\
\text { Especificidad: } 67\end{array}$ \\
\hline Yergason & $\begin{array}{l}\text { Sensibilidad: } 32 \\
\text { Especificidad:87 }\end{array}$ \\
\hline \multicolumn{2}{|c|}{ Redondo Menor } \\
\hline Prueba del redondo menor/Hornblower's sign & $\begin{array}{l}\text { Sensibilidad: } 100 \\
\text { Especificidad: } 93\end{array}$ \\
\hline
\end{tabular}

Tomado de: Gómez AJM 2014,1 Jain et al 2013,10 Fernández et al 2010.33 


\section{Conclusiones}

Existe una correlación moderada de las pruebas clínicas con el ultrasonido en la detección de LMR en forma global. Sin embargo, de forma individual, la técnica por imagen reportó más lesiones que la clínica, por lo que es un auxiliar confiable para el diagnóstico y grado de lesión.

\section{Conflicto de intereses}

Los autores declaran no tener conflicto de intereses.

\section{Bibliografía}

1. Gómez AJM. El manguito de los rotadores. Orthotips 2014; 10(3): 144-153.

2. V. Pandey, W. Jaap Willems. Rotator cuff tear: A detailed update. Asia-Pacific Journal of Sports Medicine, Arthroscopy, Rehabilitation and Technology 2015; 2(1): 1-14.

3. Juel G. Niels, Natvig Bard. Shoulder diagnoses in secondary care, a one year cohort. BMC Musculoskeletal Disorders 2014; 15(89):, 1-8.

4. Diagnóstico y tratamiento del síndrome de hombre doloroso en primer nivel de atención. México: Secretaría de Salud; 03/11/2016.

5. Virta et al. Costs of shoulder pain and resource use in primary health care: a cost of illness study in Sweden. BMC Musculoskeletal Disorders 2012; 13(17): 1-11.

6. Matthewson et al. Partial thickness rotator cuff tears: current concepts. Advances in orthopedics 2015; 1-11.

7. Greenberg L. Evalution and treatment of shoulder pain. Med Clin N Am 98 2014; 487-504.

8. Östör J. K. et al. Validation of clinical examination versus magnetic resonance imaging and arthroscopy for the detection of rotator cuff lesions. Clin Rheumatol 2013; 32: 1283-1291.

9. Chiu et al. Shoulder ultrasonography performed by orthopedic surgeons increases efficiency in diagnosis of rotator cuff tears. Journal of Orthopaedic Surgery and Research. 2017; 12(63): 1-10.

10. Jain et al. Clinical examination of the rotator cuff. PM R. Author manuscript 2014; 5(1): 1-26.

11. S. Saraya, R. El Bakry. Ultrasound: can it replace MRI in the evaluation of the rotator cuff tears?, The Egyptian Journal of Radiology and Nuclear Medicine 2016; 47: 193-201.

12. Micheroli et al. Correlation of findings in clinical and high resolution ultrasonography examinations of the painful shoulder. J Ultrason, 2015; 15: 29-44.

13. Naredo E. et al. Painful shoulder: comparison of physical examination and ultrasonographic findings. Ann Rheum Dis 2002; 61: 132-136

14. Gumina $S$. et al. The impact of aging on rotator cuff tear size. Musculoskelet Surg 2013; 97(1): S69-S72.

15. Forthomme B. et al. Shoulder isokinetic assessment: a critical analysis. Isokinetics and Exercise Science 2005; 13: 59-60.

16. Cools $M$. et al. Evaluation of isokinetic force production and associated muscle activity in the scapular rotators during a protractionretraction movement in overhead athletes with impingement symptoms. Br J Sports Med 2004; 64-68.
17. Warner et al. Patterns of flexibility, laxity, and strength in normal shoulders and shoulders with instability and impingement. Am J Sports Med. 1990; 18(4): 366-375.

18. Hughes E. et al. Normative values of agonist-antagonist shoulder strength rations of adults aged 20 to 78 years. Arch Phys Med Reh 1999; 1324-1326.

19. Noffal GJ. Isokinetic eccentric to concentric strength ratios of the shoulder rotator muscles in throwers and nonthrowers. Am J Sports Med 2003; 31(4): 537-541.

20. Treiber et al. Effect of theraband and lightweight dumbbell training on shoulder rotation torque and serve performance in college tennis players. Am J Sports Med 1998; 26(4): 510-515.

21. Dulgeroglu et al. Evaluation of Shoulder Rotational Strength in Patients with subacromial impingement syndrome using a computerized isokinetic dynamometer. Journal of Musculoskeletal Pain 2013; 21(1): 23-30.

22. Kumath $U$. et al. Correlation of clinical finding with ultrasound diagnosis of rotator cuff pathology. Research and reviews: journal of medical and health sciences 2014; 3(2):79-83.

23. Ah Kim et al. Ultrasonographic findings of painful shoulders and correlation between physical examination and ultrasonographic rotator cuff tear. Mod Rheumatol 2007; 17: 213 219.

24. Titchener et al. Comorbidities in rotator cuff disease: a casecontrol study. J Shoulder elbow surg 2014; 1-7.

25. Rechardt et al., Lifestyle and metabolic factors in relation to shoulder pain and rotator cuff tendinitis: a population-based study, BMC musculoskeletal disorders 2010; 11(165): 1-11.

26. Oliva et al. Calcific tendinopathy of rotator cuff tendons. Sports Med Arthrosc Rev 2011; 19(3): 237-243.

27. Oliva et al., Physiopathology of intratendinous calcific deposition, BMC Medicine, 2012, 10(95): 1-10.

28. Silverstein et al. Natural course of nontraumatic rotator cuff tendinitis and shoulder symptoms in a working population. Scand J Work Environ Health 2006; 32(2): 99-108.

29. Seidler et al. Work-related lesions of the supraspinatus tendon: a case-control study. Int Arch Occup Environ Health 2011; 84: 425-433.

30. Arteaga et al. Valoración clínica de la enfermedad del manguito rotador. Rehabilitación (Madr) 1998; 32: 171-180.

31. Neviaser TJ. The role of the biceps tendon in the impingement syndrome. Orthop Clin North Am 1987; 18(3): 383-386.

32. Taneja AK.et al. MRI Findings of Rotator Cuff Myotendinous Junction Injury. Am J Roentgenol 2014; 203: 406-411.

33. Fernández et al. Maniobras exploratorias del hombro doloroso .Semin Fund Esp Reumatol 2010;11(3):115-121. 